\title{
Analysis of the FliM/FliG motor protein interaction by two-hybrid mutation suppression analysis
}

Correspondence
Donna L. Marykwas
dmarykwa@csulb.edu

Received 6 November 2007

Revised 10 December 2007

Accepted 11 December 2007

\author{
Steven E. Passmore, Rithy Meas and Donna L. Marykwas
}

\author{
Department of Biological Sciences, California State University, Long Beach, Long Beach, \\ CA 90840, USA
}

\begin{abstract}
The Escherichia coli motor proteins FliM and FliG physically interact, presumably to control one or more of the functions of the bacterial flagellum clockwise/counterclockwise (CW/CCW) switch. We have previously demonstrated this interaction using the yeast two-hybrid system and have identified mutations in fliG that disrupt the interaction. Starting with the most interaction-defective of these fliG mutants, we mutagenized fliM to identify suppressor mutations that restore the FliM/ FliG two-hybrid interaction. Certain fliM suppressor mutations exhibit allele specificity. These mutations help define a FliG-interaction surface on FliM. Moreover, the pattern of suppression suggests that two distinct sites on FliG interact with FliM, perhaps with two FliM molecules in a dimer per molecule of FliG.
\end{abstract}

\section{INTRODUCTION}

Motile bacteria such as Escherichia coli continuously monitor their surroundings in pursuit of a more favourable environment and influence their own survival by modulating the direction of rotation of their flagellar rotary motors.

When the motors of a given cell rotate counterclockwise (CCW), the flagella form a coherent bundle that drives the cell steadily forwards: it runs. When they rotate clockwise (CW) the bundle flies apart and the cell tumbles. When the flagella rotate CCW once again, the cell runs in a new direction, chosen approximately at random. Runs are extended in response to positive changes in a cell's environment that are measured and compared over time. In this way, the cell executes a biased random walk and drifts in a favourable direction (reviewed by Berg, 1988).

We have adopted a molecular genetic approach to learn more about the machinery of the flagellar switch. Three proteins are known to influence switching, FliG, FliM and FliN. They appear to be localized at the base of the flagellar motor, in or near a ring known as the $\mathrm{C}$ ring (Francis et al., 1994; Khan et al., 1992) because it extends into the cytoplasm. Remarkably, FliG, FliM and FliN each possess multiple functions. They are each required for flagellar assembly and for torque generation as well as for switching (Lloyd et al., 1996; Yamaguchi et al., 1986a, b). They are

Abbreviations: CCW, counterclockwise; CW, clockwise.

Three supplementary figures, showing flim mutagenesis to isolate suppressors of fliG mutations disrupting FliM/FliG interaction, mapping of flim suppressor mutations by multifragment cloning in vivo, and an $E$. coli FliG model, a supplementary table listing primers used in this study, and the coordinates for the modelled structures of E. coli FliG and FliM, are available with the online version of this paper. expressed within an ordered hierarchy of flagellar genes (Gillen \& Hughes, 1991; Kutsukake et al., 1990), suggesting that they might also be involved in gene control.

Each switch component (FliG, FliM or FliN) can be altered to produce motors that are either overly CW-biased or overly CCW-biased and, in either case, defective for chemotaxis (Irikura et al., 1993; Magariyama et al., 1990; Sockett et al., 1992; Yamaguchi et al., 1986a, b). Switching defects of opposite bias are often compensatory but not allele-specific (Parkinson et al., 1983; Sockett et al., 1992; Yamaguchi et al., 1986a). Likewise, the interdependent localization and stability of the switch proteins (Tang et al., 1995; Tang \& Blair, 1995; Zhao et al., 1995, 1996b) can be due to either direct or indirect interactions.

Previous work (Marykwas \& Berg, 1996; Marykwas \& Passmore, 1995; Marykwas et al., 1996) has revealed direct interactions between the known components of the switch (FliG, FliM and FliN) by use of the two-hybrid system developed in yeast for the identification of interacting proteins (Chien et al., 1991; Fields \& Song, 1989). In this system, when two proteins that interact are fused to separate domains of the yeast transcription factor GAL4, interaction between the two proteins reunites the two GAL4 domains in a transcription-competent complex. Using this method, we have identified FliG/FliM, FliM/ FliM and FliM/FliN switch protein interactions, and we have shown that the switch is attached to the MS ring, the presumed rotor, via interaction between FliG and FliF (part of the MS ring). These proteins appear to interact in series, FliF with FliG with FliM with FliN. Much of what we have determined about how the switch proteins interact is consistent with the prior (Oosawa et al., 1994) and subsequent (González-Pedrajo et al., 2006; Grünenfelder et al., 2003; Kihara et al., 2000; Mathews et al., 1998; Tang 
et al., 1996; Toker et al., 1996; Toker \& Macnab, 1997) work of other research groups.

Regions of proteins important for several of these interactions have been identified by mutational analysis (Marykwas \& Berg, 1996; Marykwas et al., 1996). This included generating fliG mutants that produce proteins defective in their interaction with FliM. These mutants have revealed amino acid residues of FliG that are important for this interaction (Marykwas \& Berg, 1996). We now report that their respective mutations are genetically suppressible by compensating changes in FliM.

\section{METHODS}

Primers, plasmids and strains. Primers and strains used in this study are described in Supplementary Table S1 and Table 1, respectively. The primers were purchased from Integrated DNA Technologies. Plasmids for detecting the FliM/FliG two-hybrid interaction have been described previously (Marykwas \& Berg, 1996; Marykwas et al., 1996). Plasmid pAD-M ${ }^{\mathrm{WT}}$ expresses a fusion of the GAL4 transcription activation domain with wild-type FliM. Plasmids pBD-G $\mathrm{G}^{\mathrm{WT}}$, pBD- $\mathrm{G}^{10}, \mathrm{pBD}-\mathrm{G}^{15}, \mathrm{pBD}-\mathrm{G}^{21}$ and $\mathrm{pBD}-\mathrm{G}^{25}$ express the GAL4 DNA-binding domain fused to wild-type FliG, and to FliG mutants $\mathrm{FliG}^{\mathrm{L} 153 \mathrm{P}}$, FliG ${ }^{\mathrm{L} 225 \mathrm{P}}$, FliG ${ }^{\mathrm{L} 146 \mathrm{Q}}$ and $\mathrm{FliG}^{\mathrm{H} 155 \mathrm{P}}$, respectively. Plasmid pGAD1f (Chien et al., 1991) is the cloning vector used to construct the wild-type (Marykwas et al., 1996) and mutant (this report) $G A L 4^{A D}-$ fliM fusions. Other plasmids used in this study were pGBT9 (Clontech), pAD-FliF and pFrame2 (Marykwas et al., 1996), and pDB3 $\Delta B$ (Bartlett \& Matsumura, 1984).

Two-hybrid analysis. The methods used for two-hybrid analysis, including yeast media and culture, transformation, two-hybrid screening on indicator plates, two-hybrid quantification by $\beta$ galactosidase assays, and recovery of plasmids from yeast cells, have been described previously (Marykwas \& Berg, 1996; Marykwas et al., 1996).

Mutagenesis. We used mutagenic gap repair to generate fliM mutations that suppressed the interaction-defective phenotype of select fliG mutations, as illustrated in Supplementary Fig. S1. A pool of mutant fliM genes was generated by PCR with Taq polymerase (Promega), using plasmid pAD-M ${ }^{\mathrm{WT}}$ as template and primers oG4AD5' and oG4AD3' (Supplementary Table S1) as flanking primers. Yeast strain GGY1::171 cells bearing either $\mathrm{pBD}-\mathrm{G}^{15}$ or pBD-G ${ }^{25}$ were cotransformed with the fliM mutant pool and with linearized (BamHI-digested) pGAD1f; in addition, they were separately transformed with plasmid $\mathrm{pAD}-\mathrm{M}^{\mathrm{WT}}$ as a control.
Transformants $(1-5 \%$ of the total) that appeared more blue on indicator plates containing X-gal than the control transformants were classified as having a suppressed phenotype.

The fliM mutant plasmids were recovered from the suppressors and retested to verify the plasmid-dependence of the suppression. This involved glass bead lysis of yeast cells that had a suppressed phenotype on indicator plates, electrotransformation of this yeast lysate into $E$. coli strain GM2163 with selection for $\mathrm{Leu}^{+}, \mathrm{Amp}^{\mathrm{r}}$ transformants, purification of the fliM mutant plasmids from the transformed E. coli, transformation of these mutant plasmids into the original fli $G$ mutant yeast strain (GGY1::171::pBD-G ${ }^{15}$ or GGY1 ::171::pBD-G ${ }^{25}$ ), and phenotypic analysis (in comparison to control transformants) on indicator plates.

Genetic mapping. We mapped our fliM mutants by multifragment cloning in vivo (Marykwas \& Passmore, 1995), as illustrated in Supplementary Fig. S2. Primers specific to fliM (Supplementary Table S1) were used to amplify various parts of the mutant and wild-type $f l i M$ genes. For this purpose, the proofreading $P f u$ polymerase (Stratagene) was used. The $5^{\prime}$ one-third and two-thirds of fliM were amplified by PCR using primer oG4AD5' combined with primer oMAW2 and primer oMAW4, respectively. The $3^{\prime}$ one-third and twothirds of fliM were amplified by PCR using primer oG4AD3' combined with primer oMAW3 and primer oMAW1, respectively. Pairs of mutant and wild-type fliM fragments that together constituted full-length fliM were recombined in vivo in yeast with linearized (BamHI-digested) pGAD1f, using yeast GGY1::171 already transformed with $\mathrm{pBD}-\mathrm{G}^{\mathrm{WT}}, \mathrm{pBD}-\mathrm{G}^{15}$ or $\mathrm{pBD}-\mathrm{G}^{25}$, and the partially mutant-partially wild-type fliM chimeras were then tested directly on indicator plates containing $\mathrm{X}$-gal to assess their effects on the FliM/FliG two-hybrid interaction and thus fliG mutation suppression.

DNA sequencing. Double-stranded plasmid DNA was purified using Qiagen columns and sequenced by Davis sequencing (Davis, CA).

fliG deletion construction. We have constructed a precise deletion of the chromosomal fliG gene coding sequence (all codons of $f l i G$ ) by PCR recombination in vitro (Yolov \& Shabarova, 1990) followed by homologous recombination in vivo to exchange the wild-type with the mutant copy of the fliG gene. The primers used for the PCR recombination are listed in Supplementary Table S1. All PCR reactions were performed using $P f u$ polymerase (Stratagene) to minimize the number of introduced mutations. The sequence upstream of $f l i G$, including part of $f l i F$, was amplified using primers oFc and oF3' with plasmid pAD-F (Marykwas et al., 1996) as template. The sequence downstream of $f l i G$, including part of $f l i H$, was amplified using primers $\mathrm{oFH}^{\prime}$ and $\mathrm{oH} 3^{\prime} \mathrm{SphI}$ with plasmid $\mathrm{pDB} 3 \Delta \mathrm{B}$ (Bartlett \& Matsumura, 1984) as template. After the upstream and downstream PCR products were purified using

Table 1. Strains used in this study

\begin{tabular}{|c|c|c|c|c|}
\hline Strain & Species & Features & Purpose & Reference or source \\
\hline SJW2771 & $\begin{array}{l}\text { S. enterica serovar } \\
\text { Typhimurium }\end{array}$ & fli $^{\mathrm{MS} \rightarrow \mathrm{I}}$ start codon lost & fliG complementation & Irikura et al. (1993) \\
\hline AAEC032 & E. coli & polA12 & Allelic exchange & Blomfield et al. (1991) \\
\hline GM2163 & E. coli & leuB6 & Recovery of LEU2 plasmids & New England Biolabs \\
\hline HCB1 & E. coli & $\begin{array}{l}\text { aka AW405, wild-type for motility } \\
\text { and chemotaxis }\end{array}$ & $\begin{array}{l}\text { fliM mutant interference tests of } \\
\text { negative dominance }\end{array}$ & Armstrong et al. (1967) \\
\hline TPD11 & E. coli & fliG null, isogenic to HCB1 & fliG complementation & This study \\
\hline
\end{tabular}


QIAquick PCR purification columns (Qiagen), the two pieces were joined in a third PCR reaction using primers oFc and oH3'SphI. The resulting PCR product includes a precise deletion of the fliG ORF flanked by upstream and downstream DNA. After an additional purification step, the final PCR product was inserted by blunt-end ligation into plasmid pGBT9 digested with SmaI. The resulting plasmid, pGBT9-'F $\Delta \mathrm{GH}$ ', was sequenced at both plasmid/insert cloning junctions and across the deletion for verification. The plasmid was integrated into the chromosome of E. coli strain AAEC032 (Blomfield et al., 1991), which contains a temperaturesensitive polA gene product, for the purpose of allelic exchange. Plasmid integration occurs at the non-permissive temperature by imposing drug selection for plasmid maintenance, and homologous de-integration and plasmid loss occur at the permissive temperature after relief of drug selection. After AAEC032 $\mathrm{fli}$ G was constructed, the now chromosomal fliG deletion was verified by PCR. We determined that the non-motile phenotype could be complemented by wild-type fliG on a plasmid, prior to transferring the deletion into the desired genetic background, isogenic to HCB1, by a series of P1 transductions using bacteriophage P1vir (Miller, 1992). The complementation was $60-70 \%$ of wild-type based on swarm size, and $100 \%$ based on tethered motor speed. This fliG null allele does not gene convert when wild-type $f l i G$ is introduced back into E. coli on a plasmid, and, based on the complementation described above, it is non-polar, even though $\mathrm{fliH}$ is presumably expressed using the fliG ribosome-binding site.

Motility tests. Swarm plates included medium containing $1 \%(\mathrm{w} / \mathrm{v})$ tryptone and $0.5 \%(\mathrm{w} / \mathrm{v}) \mathrm{NaCl}($ tryptone broth, $\mathrm{TB})$ and $0.3 \%(\mathrm{w} / \mathrm{v})$ agar. Ampicillin, at a final concentration of $100 \mu \mathrm{g} \mathrm{ml} \mathrm{m}^{-1}$, was included, when necessary, to maintain plasmid selection. The medium is semi-solid, so motile, chemotactic bacteria can swim through it to establish large colonies with concentric rings of migrating cells. Swarm plates were inoculated with cells taken, using sterile toothpicks, from colonies newly grown overnight. The plates were incubated at $30{ }^{\circ} \mathrm{C}$ in an enclosed plastic box kept humid by the inclusion of water in a beaker.

Bacteria grown in $\mathrm{TB}$ at $30{ }^{\circ} \mathrm{C}$ were examined microscopically to assess their swimming behaviour. In addition, TB-grown cultures of bacteria were stained to permit microscopic visualization of flagella, following published procedures (Heimbrook et al., 1989).

Tethering assays. Anti-flagellin antibodies (produced for us by Cocalico) were bound to acid-washed glass coverslips by spotting $1 \mu \mathrm{l}$ antibody solution onto a glass coverslip, which was then dried in a humid, cool $\left(4{ }^{\circ} \mathrm{C}\right)$ environment. Before use, the antibody-treated coverslips were washed with sterile water and then with sterile TB. Flagella were sheared by repeatedly passing cells through a 24-gauge needle. Sheared cells were similarly washed and spotted onto the coverslips, then inverted onto glass depression slides. The rotation of tethered cells (Silverman \& Simon, 1974) was observed using an Olympus CX41 microscope with a video camera attachment. Images were captured using an EchoFX InterView Lite capture device and recorded on an iMAC G3 computer. Movies were played back one frame at a time to measure the speed and direction of rotation.

Protein structure modelling. We used the automated proteinmodelling server SWISS-MODEL (Schwede et al., 2003) to generate a theoretical model of the E. coli FliG protein (residues 114-323), based on the Thermotoga maritima structural information (Brown et al., 2002; Lloyd et al., 1999). SWISS-MODEL uses the program ProModII (version 3.70) for modelling and the program GROMOS96 (Scott et al., 1999) for energy minimization. The coordinates for the modelled structure of E. coli FliG are provided as a supplement (FliGEcoli.pdf). We have used the program SWISS-PdbViewer (http://www.expasy.org/spdbv/) to view and manipulate these structural models.

We used the same programs and techniques to model the E. coli FliM protein (residues 43-228) based on the T. maritima FliM structure (Park et al., 2006). The coordinates for the modelled structure of $E$. coli FliM are also provided as a supplement (FliMEcoli.pdf). Our model based on T. maritima FliM is comparable to an earlier model provided by Dr David Blair, The University of Utah, that is based on the structure of the related protein CheC (Park et al., 2004) and which allowed us to begin this structural analysis. We used this information and the above protein structure modelling software to generate a composite model of the FliM/FliG interaction interface, based on the locations and nature of the amino acid substitutions that suppressed defective interaction in the fliM and fliG mutants.

\section{RESULTS}

\section{FliG and the FliG/FliM interaction}

The FliG/FliM interaction has been demonstrated using the yeast two-hybrid system. FliG fused to the GAL4 DNAbinding domain interacts with FliM fused to the GAL4 activation domain (Marykwas et al., 1996). Interactiondefective fli $G$ mutants (defective in their interaction with FliM) have been isolated, mapped and identified. They map to the middle third of FliG, a region important for switching (Irikura et al., 1993; Togashi et al., 1997), in two subclusters (Marykwas \& Berg, 1996). The structure of FliG, albeit from T. maritima, is now partially solved (Brown et al., 2002; Lloyd et al., 1999). We have modelled the structure of E. coli FliG, using the structural coordinates of T. maritima FliG (Fig. 1). Fifteen of our previously identified fliG mutations that result in a FliMinteraction-defective phenotype affect amino acid residues within the modelled domain.

The interaction-defective $f l i G^{15}$ (L225P) and $f_{l i G^{25}}$ (H155P) substitutions lie in different subclusters and represent two separate potential FliM-binding sites along the primary amino acid sequence of FliG (Marykwas \& Berg, 1996). They are both represented within the modelled structure of the protein. Fig. 1 shows the E. coli FliG model with the positions affected by these substitutions highlighted. These two fliG mutant proteins also confer different motility phenotypes. A fliG null strain of Salmonella enterica serovar Typhimurium (SJW2771; Irikura et al., 1993) expressing fli $G^{15}$ from a plasmid has paralysed flagella (the $\mathrm{Fla}^{+}, \mathrm{Mot}^{-}$phenotype). However, expression of $f l i G^{25}$ yields cells that are flagellate and motile but tumbly (the $\mathrm{Fla}^{+}, \mathrm{Mot}^{+}, \mathrm{Che}^{-}$phenotype). For these reasons, and because fli ${ }^{15}$ and $f l i G^{25}$ are two of the more severe interaction-defective mutants, we chose them for suppression analysis. We also constructed and tested these mutants in a fliG-null strain of E. coli (see Methods). The phenotypes of these mutants, and two others described later, are summarized in Table 2 and illustrated in Fig. 2. The tumbly nature of $f l i G^{25}$ (and $f l i G^{10}$ ) was exhibited by swimming cells of $E$. coli and $S$. enterica serovar Typhimurium and verified by tethering assays with E. coli. 


\begin{tabular}{ll|cccccc} 
FliG & 1 & 50 & 100 & 150 & 200 & 250 & 300 \\
E. coli & 531 &
\end{tabular}

FliM-binding defective

T. maritima FliG

Structure

E. coli FliG Model

Defective

Front
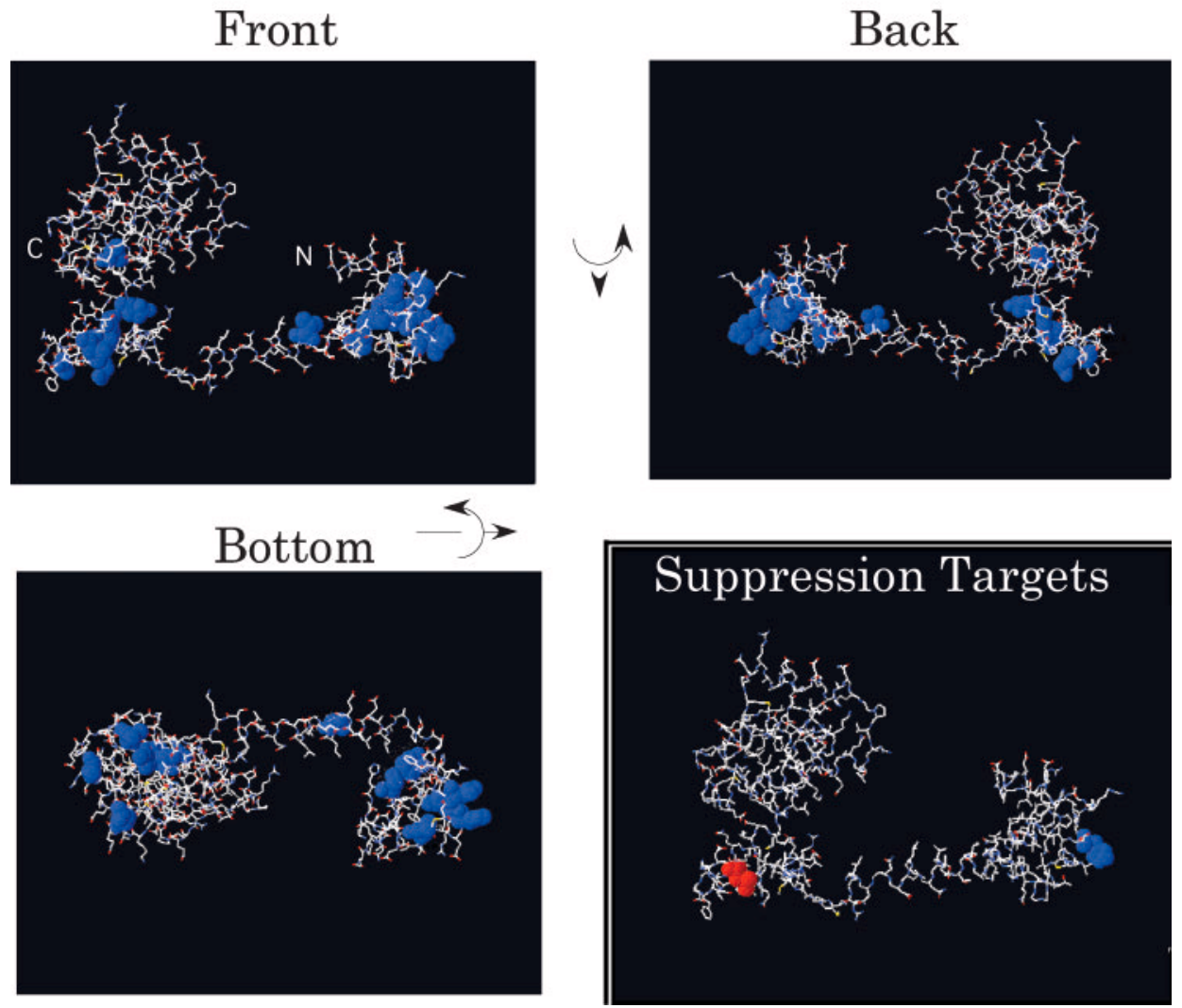

Fig. 1. E. coli FliG model and positions of interaction-defective amino acid substitutions. The top bar represents the $E$. coli FliG protein. The FliG amino acid substitutions that impair the FliM/FliG interaction (Marykwas \& Berg, 1996) are indicated in blue, both on the linear depiction of the primary sequence and on the structure. They include M113T, I129L, T132S, V135A, L146Q, L153P, H155P, V157A, L159S, V178L, Q210R, A217T, L225P, I229T, E232V and V312E. The light-grey bar indicates the extent of structural information that is available for T. maritima FliG (Brown et al., 2002; Lloyd et al., 1999). The black bar indicates the extent of the E. coli FliG model based on the T. maritima structure. M113T is off the structure. The substitutions from I129L to L159S are present in the high-affinity FliM-binding site (site 1) that we predict and describe in the Discussion. They surround the EHPQR motif. V178L is located in the linker region. The substitutions from Q210R to E232V are present in the low-affinity FliM-binding site (site 2), adjacent to the hydrophobic patch, including two that overlap. V312E and E232V are near each other on the structure, so that $\mathrm{V} 312 \mathrm{E}$ is close to but not part of site 2. The bottom-right panel indicates the positions on FliG of two amino acid substitutions that disrupt the FliM/FliG interaction, chosen as the starting point for the fliM suppression analysis; L225P is indicated in red and H155P in blue.

The mutants that were tumbly swimmers appeared to reverse more frequently than cells harbouring $\mathrm{pBD}-\mathrm{G}^{\mathrm{wt}}$ expressing the wild-type FliG fusion. The mutants that were $\mathrm{Fla}^{-}$in $E$. coli were non-tetherable, as would be expected if they indeed lacked flagella.

\section{fliM-dependent suppression of fliG alleles}

We hypothesized that if FliG residues L225P and H155P are important for interaction with FliM, then we should be able to isolate fliM mutants that restore the FliM/FliG 
Table 2. fliG mutant phenotypes

\begin{tabular}{|c|c|c|c|}
\hline Allele & $\begin{array}{l}\text { Amino acid } \\
\text { substitution }\end{array}$ & $\begin{array}{l}\text { S. enterica serovar } \\
\text { Typhimurium } \\
\text { phenotype }\end{array}$ & E. coli phenotype \\
\hline 10 & L153P & $\begin{array}{l}\mathrm{Fla}^{+}, \mathrm{Mot}^{+}, \\
\mathrm{Che}^{-}, \text {tumbly }\end{array}$ & $\begin{array}{l}\mathrm{Fla}^{+}, \mathrm{Mot}^{+}, \mathrm{Che}^{-}, \\
\text {tumbly, switches often }\end{array}$ \\
\hline 15 & L225P & $\mathrm{Fla}^{+}, \mathrm{Mot}^{-}$ & $\mathrm{Fla}^{-}$ \\
\hline 21 & L146Q & $\mathrm{Fla}^{+}, \mathrm{Mot}^{-}$ & $\mathrm{Fla}^{-}$ \\
\hline 25 & $\mathrm{H} 155 \mathrm{P}$ & $\begin{array}{l}\mathrm{Fla}^{+}, \mathrm{Mot}^{+}, \\
\mathrm{Che}^{-} \text {, tumbly }\end{array}$ & $\begin{array}{l}\mathrm{Fla}^{+}, \mathrm{Mot}^{+}, \mathrm{Che}^{-}, \\
\text {tumbly, switches often }\end{array}$ \\
\hline
\end{tabular}

interaction. To mutagenize $f l i M$, we followed a mutagenic gap repair protocol, as described in Methods. Mutant fliM fusion genes (that fuse wild-type GAL4 ${ }^{\mathrm{AD}}$ in-frame with mutant FliM) were generated in yeast in vivo and scored directly for their ability to suppress the two-hybrid interaction defects of these two fliG mutant proteins, encoded on plasmids $\mathrm{pBD}-\mathrm{G}^{15}$ or $\mathrm{pBD}-\mathrm{G}^{25}$, respectively. The newly generated fliM mutant plasmids were recovered from the suppressors, and each one was retested to verify the plasmid-dependence of the suppression, yielding seven suppressors of $f l i G^{15}$ and 14 suppressors of $f l i G^{25}$.

\section{Mutation mapping and identification}

Each plasmid-borne fliM mutant was sequenced to locate the positions of mutations within the fliM DNA. Ten of the $21 \mathrm{fliM}$ mutants each harboured a single mutation resulting in an amino acid substitution. The remaining 11 mutants

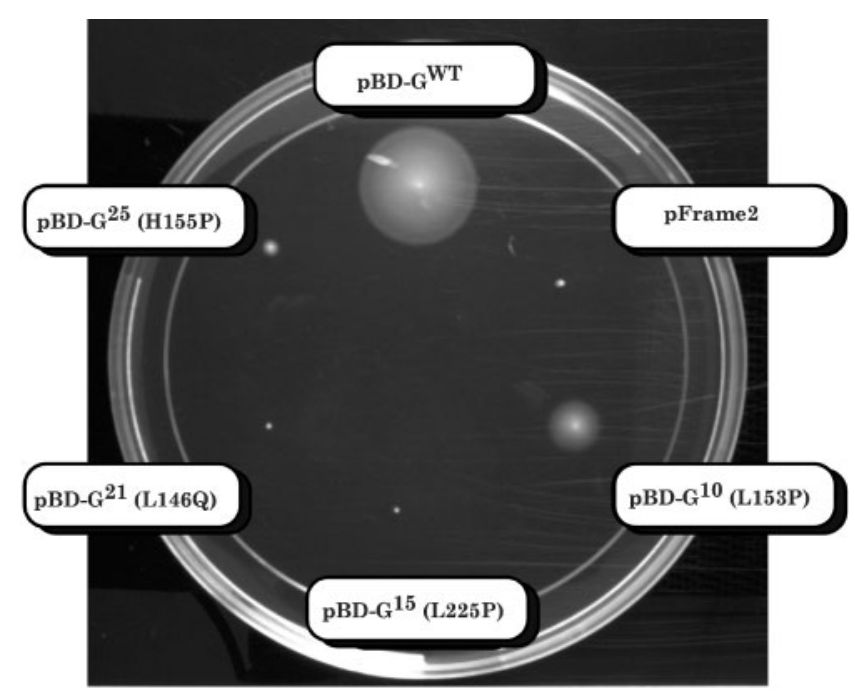

Fig. 2. Motility of interaction-defective fliG mutants on swarm plates. A fliG null strain of $E$. coli was transformed with plasmidborne fliG mutations and tested for motility on ampicillin-containing swarm agar. The plasmid pFrame2 is a cloning vector that does not express FliG. Wild-type FliG fused in-frame with GAL4 DNAbinding domain complements the motility defect of this strain. each bore more than one mutation. Thus, for each mutant, it was essential to determine which of the fliM mutations was responsible for restoring the interaction with mutant FliG. To do this, we mapped the flim mutations by multifragment cloning in vivo (Marykwas \& Passmore, 1995; Supplementary Fig. S2), separating them by in vivo subcloning, and then testing them for their effects on the FliM/FliG two-hybrid interaction. We thus assigned responsibility to one or more mutations, even when multiple mutations were present, for all but four mutants; those four had mutations that were too numerous or too closely linked to be sorted. Table 3 lists the identity of the fliM amino acid substitutions responsible for fliG suppression, including the number of times each fliM mutation was independently isolated. One of our $21 \mathrm{fliM}$ mutants had both T139I and T149I, and another had both N101S and T149I. Each of these substitutions was also identified on its own in independent suppressors. In addition to those mutations responsible for suppression, we identified 23 mutations spread throughout the gene that do not inhibit binding (results not shown).

\section{Allele specificity}

Although each fliM mutant was isolated as a suppressor of either $\mathrm{fli}^{15}$ (L225P) or $\mathrm{fliG}^{25}$ (H155P), each mutant was further tested for its effects on the interaction with wildtype FliG and selected fliG mutants, including $\mathrm{fli}^{10}$ (L153P) and $f l i G^{21}$ (L146Q) as well as $f l i G^{15}$ (L225P) and $\mathrm{fli}^{25}$ (H155P). The motility phenotypes of these four $f l i G$ mutants are summarized in Table 2. The FliM/FliG twohybrid interaction was measured qualitatively on indicator plates containing X-Gal and, for some, quantitatively by in vitro assays of $\beta$-galactosidase activity (Table 4). The fliM alleles were expressed as GAL4 ${ }^{\mathrm{AD}}$ fusions. The fliG alleles were expressed as $\mathrm{GAL4}^{\mathrm{BD}}$ fusions. The degree of interaction was scored on a scale of 1 to 5 , based on the blueness of the yeast colonies co-expressing the mutant two-hybrid fusions relative to the dark blue (scored as 5) phenotype conferred by the two wild-type fusions. The degree of interaction was scored for the original 21 plasmid-borne fliM mutants, before the identity of the fliM mutations was known. A sample was chosen for

Table 3. fliM mutations that suppress fliG mutations

\begin{tabular}{|lccc|}
\hline fliG & FliG amino acid & FliM suppressor amino & Number of \\
alle & substitution & acid substitution & isolates \\
\hline fliG $^{15}$ & L225P & N129I & 1 \\
& & H148R & 2 \\
$\mathrm{fliG}^{25}$ & \multirow{2}{*}{ H155P } & M184K & 1 \\
& & N101S & 2 \\
& & N129I & 2 \\
& & T139I & 2 \\
& & H148R & 4 \\
& & T149I & 5 \\
\hline
\end{tabular}


Table 4. Two-hybrid interaction data reveal allele-specific suppression of FliM/FliG interaction

Bold type indicates suppression. Blueness was judged by eye and labelled from 5 (most blue) to 1 (least blue).

\begin{tabular}{|lccccc|}
\hline \multirow{2}{*}{$\begin{array}{l}\text { FliM amino acid } \\
\text { substitution }\end{array}$} & \multicolumn{5}{c|}{ FliG amino acid substitution } \\
\cline { 2 - 6 } & WT & L146Q & L153P & H155P & L225P \\
\hline WT & $5^{*}$ & $1 \dagger$ & $2 \ddagger$ & 1 & 2 \\
N101S & 5 & 1 & 2 & $\mathbf{2}$ & 2 \\
N129I & 5 & 1 & $4 \|$ & $\mathbf{3 9}$ & $\mathbf{4}$ \\
T139I & 5 & 1 & $\mathbf{3}$ & $\mathbf{2}$ & $\mathbf{3}$ \\
H148R & 5 & 1 & $\mathbf{4}$ & $\mathbf{3}$ & $\mathbf{4}$ \\
T149I & 5 & $\mathbf{2}$ & $\mathbf{5}$ & $\mathbf{4}$ & $\mathbf{5}$ \\
M184K & 5 & 1 & 2 & 1 & $3 \S$ \\
\hline
\end{tabular}

$\star 235 \pm 19$ units $\beta$-galactosidase (mg protein $)^{-1}$ (mean \pm SEM).

$\dagger 1.4 \pm 0.02$ units $\mathrm{mg}^{-1}$.

$\ddagger 8 \pm 2.3$ units $\mathrm{mg}^{-1}$.

§Allele-specific suppression.

$1142 \pm 0.5$ units $\mathrm{mg}^{-1}$.

$18 \pm 1.6$ units $\mathrm{mg}^{-1}$.

quantification by in vitro $\beta$-galactosidase assays, performed in duplicate. The qualitative and quantitative data agreed, but the correspondence was non-linear. These activities are consistent with values reported by others for biologically relevant protein-protein interactions (Estojak et al., 1995).

None of the amino acid substitutions caused by the fliM mutations significantly impaired FliM binding to wild-type FliG (Table 4). Thus, the suppressor-dependent changes to the FliM protein restore interaction with defective FliG without disturbing the wild-type protein-protein interaction.

FliM substitution N101S appears to be specific for $\mathrm{fliG}^{25}$ (H155P). FliM substitution M184K appears to be specific for $\mathrm{fliG}^{15}$ (L225P). FliM substitutions N129I, T139I and H148R did not suppress fliG $^{21}$ (L146Q), but did suppress the other mutant alleles tested. Interestingly, mutations resulting in FliM substitutions H148R and N129I were each independently isolated as suppressors of both $\mathrm{fli}^{15}$ (L225P) and $f_{l i G^{25}}(\mathrm{H} 155 \mathrm{P})$, in agreement with the tests of allele specificity. The FliM substitution T149I caused non-specific suppression, since all interactions were enhanced.

\section{Tests of dominance in E. coli}

We tested the plasmid-borne fliM mutations that suppress defective FliG binding to see whether they exert dominant negative effects on the motility of wild-type E. coli. Five of the mutations exerted dominant effects on swimming behaviour (switching frequency and switching bias, Table 5). Interestingly, although the FliM amino acid substitutions M184K, H148R, T149I, N129I and N101S
Table 5. fliM motility phenotypes in a wild-type background

All alleles were tested in an HCB1 background.

\begin{tabular}{|ll|}
\hline $\begin{array}{l}\text { Amino acid } \\
\text { substitution }\end{array}$ & \multicolumn{1}{c|}{ Motility phenotype } \\
\hline $\mathrm{WT}^{\star}$ & $\mathrm{Fla}^{+}, \mathrm{Mot}^{+}$, wild-type runs and tumbles \\
N101S & $\mathrm{Fla}^{+}, \mathrm{Mot}^{+}$, smooth \\
N129I & $\mathrm{Fla}^{+}, \mathrm{Mot}^{+}$, smooth, seldom switches \\
T139I & $\mathrm{Fla}^{+}, \mathrm{Mot}^{+}$, wild-type runs and tumbles \\
H148R & $\mathrm{Fla}^{+}, \mathrm{Mot}^{+}$, smooth, seldom switches \\
T149I & $\mathrm{Fla}^{+}, \mathrm{Mot}^{+}$, tumbly \\
M184K & $\mathrm{Fla}^{+}, \mathrm{Mot}^{+}$, tumbly \\
\hline
\end{tabular}

${ }^{*}$ Wild-type FliM.

each increased the affinity of FliM for mutant FliG protein, some resulted in overly tumbly swimming, while others resulted in overly smooth swimming when expressed in wild-type E. coli.

\section{Structural modelling of FliM, FliG and the FliM/ FliG interaction}

To gain a better understanding of how fliM suppressor mutations can lead to restoration of the FliM/FliG interaction with mutant FliG protein, we used SWISSMODEL (see Methods) to see where the amino acid residues involved in suppression lie on the 3D structures of FliM (Fig. 3) and FliG (Fig. 1). Our previously identified interaction-disrupting fliG mutations primarily affect residues in two clusters on the bottom surface of the extended 'arm' of the C-terminal half of FliG (residues 129-178 and 210-232), suggesting a likely interaction surface for FliM binding. Of the substitutions caused by fliG mutations tested in this study, L146Q involves an amino acid residue that is partially buried in the FliG structure, explaining why it is relatively difficult to suppress, whereas L153P, H155P and L225P involve residues that are exposed. Although the conserved FliG EHPQR motif and the hydrophobic patch described by Brown et al. $(2002,2007)$ contribute to the FliM-binding site(s), our interaction-disrupting fliG mutations mostly affect residues near but not within these conserved groups of residues. The exceptions are two interaction-disrupting mutations that alter residues 225 and 229 of the hydrophobic patch. The mutation affecting residue 225 is one that we chose to suppress. Interestingly, FliG (V196A) is a silent substitution within the hydrophobic patch that neither increases nor decreases the FliM/FliG two-hybrid interaction (Marykwas \& Berg, 1996), supporting the importance of the hydrophobic nature of this residue.

The suppressor mutations affect residues that are near each other on the top surface of FliM (Fig. 3). One affected residue is not included on the FliM model because it is in a loop that cannot be modelled accurately. However, both 


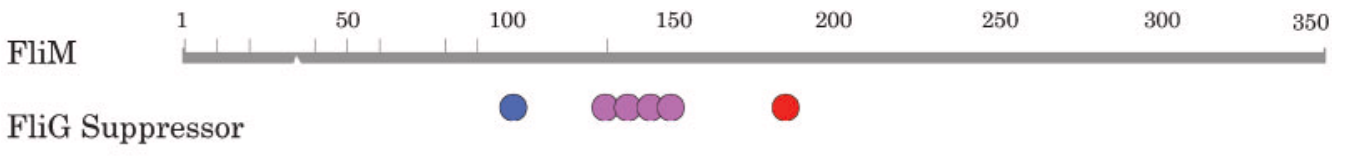

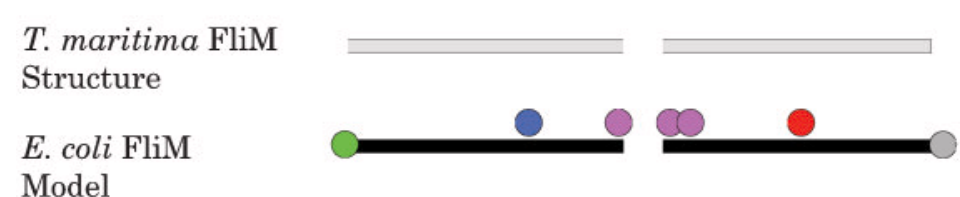

Model

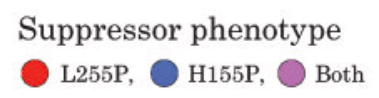

Fig. 3. FliM amino acid substitutions that suppress binding-defective FliG amino acid substitutions. The top bar represents the FliM protein. The amino acid substitution that specifically suppresses FliG (H155P) is indicated in blue. The one that suppresses FliG (L225P) is in red. Those that suppress both FliG amino acid substitutions are in purple. The FliM N and C termini are in green and grey, respectively. The light-grey bar indicates the extent of structural information that is available for the T. maritima FliM protein (Park et al., 2006). The black bar indicates the extent of the E. coli FliM model based on the T. maritima FliM structure.

ends of this loop and thus the affected residue contained therein are also located on or near this top surface.

\section{DISCUSSION}

\section{FliM/FliG interactions}

fliM mutations that suppress interaction-disrupting fliG mutations have been isolated, mapped and further characterized by DNA sequencing. Consistent with twohybrid assays indicating that neither the $\mathrm{N}$-terminal 38 (data not shown) nor the C-terminal 52 (Marykwas et al., 1996) amino acids of FliM are required for FliM/FliG interaction, the suppressors map to the middle third of fliM. Suppression by certain fliM mutations appears to be allele-specific. In other words, specific changes in FliM (fused to the GAL4 activation domain) restore the interaction disrupted by specific changes in FliG (fused to the GAL4 binding domain). Our fliM mutants were identified on the basis of their restored FliM/FliG interaction, independent of their switch bias and the complications that arise from using motility-based screens (Parkinson et al., 1983; Sockett et al., 1992; Yamaguchi et al., 1986a). Allele specificity (Jarvik \& Botstein, 1975), particularly in the context of a direct binding assay such as the two-hybrid system (Brent \& Finley, 1997), suggests specific interaction between the two proteins, perhaps involving those residues altered in the suppression or nearby residues whose positions may have been indirectly affected. This interpretation is consistent with the location of the 'interacting' residues on the structural model of the FliM/FliG interaction. Suppression by other fliM mutations appears to be allele-restricted, suppressing a subset of $f l i G$ mutations (Sandrock et al., 1997; Sujatha \& Chatterji, 2000), also consistent with the structural model.

Most of the fliM mutations identified using the two-hybrid interaction assay also affect switching bias and/or switching frequency, as expected if the FliM/FliG interaction interface is involved in these aspects of switch-complex function. We note that among the allele-specific and allele-restricted fliM mutations described in this paper, the two that yield the highest two-hybrid affinity for FliG encode FliM amino acid substitutions N129I and H148R. Since they suppress amino acid substitutions in different lobes of FliG, we imagine that they restore the interaction with FliG by increasing the affinity of FliM for both of the FliG binding 
sites. Furthermore, when the mutant FliM proteins bearing the N129I and H148R amino acid substitutions are expressed in wild-type E. coli, the cells swim smoothly with very few directional changes, suggesting that these particular mutant FliM proteins favour a CCW (smooth) state.

The fact that the two-hybrid suppression screen is not dependent upon CW/CCW switch bias compensation is illustrated, for example, by our finding that FliG amino acid substitutions L153P and H155P, that yield tumbly swimming when expressed in E. coli, can interact in the two-hybrid system with mutant FliM proteins that yield opposite (dominant smooth or dominant tumbly) swimming phenotypes.

\section{Model for motor structure/function}

Fig. 4 summarizes our interaction data in the context of existing structural information. The structure of the FliM/ FliG interaction is shown next to a processed electron micrograph image of the basal body/C-ring complex and with cartoons depicting two-hybrid switch protein interactions, including a view of these interactions in the motor in cross section. This is not a true atomic model of the interaction interface; the structures are intentionally spread out to permit visualization of the regions involved in pairwise interactions, which are highlighted in corresponding colours. The pattern of suppression between fliG and fliM mutations and the locations of interacting FliG and FliM residues suggest that two spatially distinct domains forming the bottom of FliG are separated by an extended arm and interact with a binding surface on the top of FliM. Based on this model of the interaction interface, it is clear that the two nearby FliM residues identified in allelespecific interactions cannot interact with FliG simultaneously, since the position FliM must adopt to accommodate one interaction excludes the second interaction. Additional FliM residues (coloured purple in Fig. 3) can be altered to suppress multiple fliG mutations with restricted specificity or non-specifically. This 'site' on the top of FliM may interact with the bottom of the FliG linker arm, wherein we did find a single interaction-defective amino acid substitution (V178L; Marykwas \& Berg, 1996). Alternatively, the 'inside' location of the FliM residues involved in allele-restricted suppression might enhance FliM/FliM interactions and thus indirectly enhance the FliM/FliG interaction. Our findings of multiple FliGbinding sites on FliM, also proposed by Brown et al. (2007), are in agreement with an analysis of fliM deletions and linker insertions that suggested the possible existence of multiple binding sites for FliG (Mathews et al., 1998).

\section{Implications of the FliM/FliG structure}

The structural model and the positions of allele-specific suppressors suggest the existence of two FliM/FliG interactions that cannot be accommodated by a single FliM.

To account for this, we suggest a model in which the occupancy of two FliM-binding sites can vary. In this model, one of the FliG sites has a higher affinity for FliM and thus a greater occupancy than the second
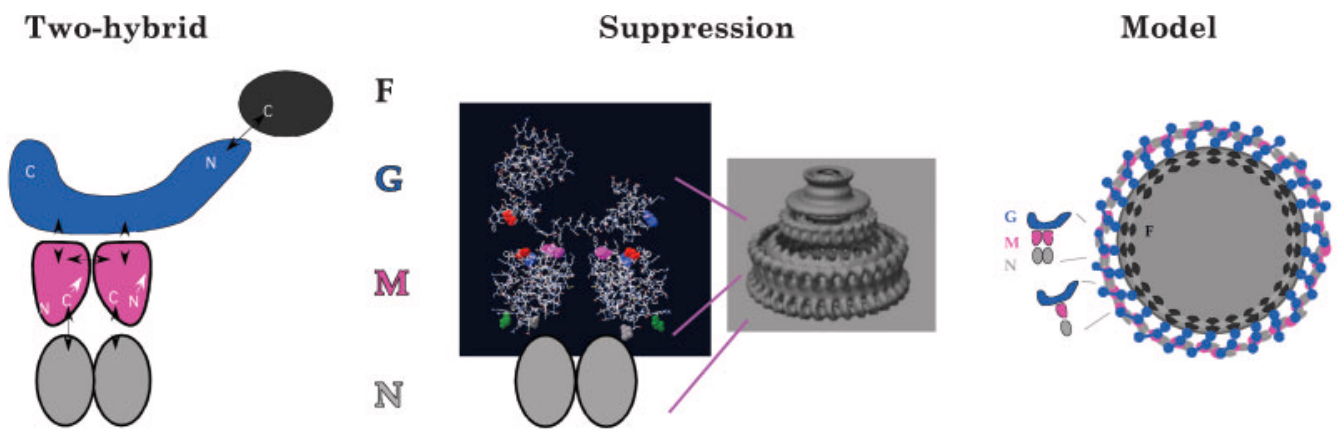

Fig. 4. Model summarizing the genetic suppression data superimposed on the FliM/FliG structure, and two-hybrid interactions of FliF (black), FliG (blue), FliM (pink) and FliN (grey). The image of the basal body/C-ring complex (Thomas et al., 2006) is used with the permission of David DeRosier. The 'interacting' residues of FliG and FliM, involved in allele-specific suppression, are colour-coded as in previous figures. The $\mathrm{N}$ and $\mathrm{C}$ termini of proteins are indicated. The double-headed arrows represent the interactions identified in this paper and in previous work in our laboratory (Marykwas \& Berg, 1996; Marykwas et al., 1996). The cross-section illustrates our view of how the MS ring with 26-fold symmetry can accommodate the C ring with 34 -fold symmetry. The 26 FliG interact with MS-ring protein FliF. One-third of FliG molecules in the motor are fully occupied with two FliM; the remaining FliG bind only one FliM, at the high-affinity binding site. This yields $26 \mathrm{FliG}: 34 \mathrm{FliM}$. Although not drawn as such, FliN probably binds as a tetramer (Brown et al., 2005). Each molecule of FliG in the model is represented by three connected blue spheres. One sphere represents the N-terminal domain of FliG interacting with FliF; the central sphere represents the central domain of FliG that includes 'site 1' bound to FliM; the remaining sphere represents the C-terminal motor domain of FliG, including 'site 2' only partially occupied by FliM. 
FliM-binding site of FliG. We further suggest that FliM can bind either the FliG high-affinity site as a monomer or both the high- and low-affinity sites as a dimer. The FliM/ FliM interaction has been analysed using the yeast twohybrid system, which reveals the existence of two binding sites within FliM for FliM self-association, one within or including the C-terminal 52 amino acids, and one elsewhere (Marykwas et al., 1996). Genetic and behavioural analysis of cells harbouring a series of scanning fliM deletions likewise suggests two FliM/FliM binding sites, one including residues $51-100$, the other including residues 261-320 near the C terminus (Toker et al., 1996). Moreover, FliM proteins bearing introduced Cys residues can be cross-linked in vivo into dimers and higher-order multimers, involving residues consistent with two binding sites (Park et al., 2006). The FliM/FliM interface (Fig. 4) contains residues 57-77, consistent with the location suggested by the above-mentioned studies (Marykwas et al., 1996; Park et al., 2006; Toker et al., 1996).

Based on genetic data reported here, site 1 includes FliG residue 155, which is involved in the allele-specific interaction between FliG (H155P) and FliM (N101S). Site 2 includes FliG residue 225, which is involved in the allelespecific interaction between FliG (L225P) and FliM (M184K). Residue 155 is closer to the FliG $\mathrm{N}$ terminus and thus assumes a position on the basal body/C-ring complex that is more interior than that of residue 225 . Thus, site 1 is the inner interaction site and site 2 is the outer interaction site. If the two FliG sites bind FliM independently, we would expect either mutation to reduce the FliM/FliG interaction to at most $50 \%$ of the wild-type interaction. However, the mutations affecting sites 1 and 2 reduce the interaction to different extents, to $\sim 0.4$ and $8 \%$ of the wild-type interaction, respectively (Marykwas \& Berg, 1996). This suggests cooperative binding of FliM to FliG. In addition, this identifies site 1 at the inner location involving FliG residue 155 as the higher-affinity site and site 2 at the outer location involving FliG residue 225 as the lower-affinity site.

Null mutants of fliG and fliM exhibit the non-flagellate phenotype. We would expect the high-occupancy site, the one subject to a more severe interaction defect, to be required for flagellum assembly, as it is. Indeed, both the high- and the low-occupancy sites are involved in flagellum assembly; FliG (L146Q) at site 1 and FliG (L225P) at site 2 are both $\mathrm{Fla}^{-}$(Table 2). Site 1 is also involved in CW/CCW switching (H155P causes defective switching; Table 2), and the suppressor mutant FliM (N101S), which is specific for FliG (H155P), affects a FliM residue that can also be substituted to become switch-biased. Although the site 2 mutant that we tested was not switch-defective, site 2 is involved in switching as well (Brown et al., 2007). It may not be possible to assign priority of function to the individual switch proteins and their interactions, since all three act together as part of a complex to control flagellum assembly and motor rotation as well as switch bias.

\section{Stoichiometry and symmetry}

Much work has been done to determine the precise number of molecules of each switch protein per flagellar basal body switch complex (Francis et al., 1994; Zhao et al., 1996a, b). Two studies have found that the MS ring contains an average of 26 interacting subunits of FliF (Jones et al., 1990; Sosinsky et al., 1992), and a recent analysis of the 3D structure of a CW-locked motor revealed a 24-26-fold M-ring symmetry (Thomas et al., 2006). FliG interacts with FliF (Francis et al., 1992; Grünenfelder et al., 2003; Kihara et al., 2000; Marykwas et al., 1996; Oosawa et al., 1994; Thomas et al., 2001) and constitutes either the extended MS-ring (Francis et al., 1994; Zhao et al., 1996b) or inner-C-ring substructure (Khan et al., 1998). The number of FliG subunits in the motor equals (Francis et al., 1992; Oosawa et al., 1994) or exceeds (Zhao et al., 1996a, b) the number of FliF subunits. Careful attempts at measuring the FliG and FliM content of purified basal body switch complexes suggest $41 \pm 10$ FliG and $37 \pm 13$ FliM per flagellum (Zhao et al., 1996a, b). Although these measurements have been used to argue for an equimolar presence of FliG and FliM in the motor, it is possible that the ratio is not $1: 1$ but might vary from $2: 1$ to $1: 1.5$.

Native C-rings exhibit a 33-35-fold rotational symmetry (Khan et al., 1998; Thomas et al., 1999). CW-locked Crings similarly exhibit a 32-36-fold outer symmetry (Thomas et al., 2006). Studies in which Salmonella MSC rings were overexpressed in non-motile $E$. coli revealed that MSC ring complexes can have MS-rings of 26-fold symmetry attached to C-rings with anywhere from 31- to 38-fold symmetry (Young et al., 2003). Inner features of the C-ring exhibit symmetry similar to that of the M-ring (Thomas et al., 2006). If FliG is part of the extended MSring, as suggested by some structural studies (Francis et al., 1994; Suzuki et al., 2004; Zhao et al., 1996b), then it is likely to share the same 26 -fold rotational symmetry and subunit number as the MS-ring. FliM and FliN, the structural proteins of the larger C-ring (Francis et al., 1994; Zhao et al., 1996a, b), would then be expected to be present in larger numbers. Indeed, FliN is the most abundant switch protein, estimated at 111 FliN molecules per flagellum (Zhao et al., 1996b). Brown et al. (2005) found that $E$. coli FliN forms stable tetramers in vitro.

How does a ring structure with 26-fold symmetry interdigitate with a larger ring structure of typically $34-$ fold symmetry? While the 34-fold symmetry of the C-ring might include contributions from additional proteins that interact with the switch complex, such as FliH and FliI (González-Pedrajo et al., 2006), the 34 -fold symmetry can be accommodated by this model of the FliM/FliG interaction (two distinct sites on FliG with different affinities for FliM) by envisioning that the affinities are such that two FliM-binding sites on FliG are bound by two FliM on approximately one-third of the FliG molecules and by one FliM on the other two-thirds. For each FliG, there would be 1.3 FliM, or 26 FliG to 34 FliM to $x$ FliN. This is 
consistent with the observed distribution of C-ring symmetries when individual motors are examined (Khan et al., 1998; Thomas et al., 1999, 2006; Young et al., 2003).

The recent paper by Brown et al. (2007), describing a mutational analysis targeted to residues on the surface of the FliG structure, also supports the existence of two FliMbinding sites. Those authors constructed mutations predicted to alter the surface of FliG, and then tested for impaired FliM binding, while our original collection of interaction-defective fliG mutations was identified after mutagenizing the entire fliG gene. Likewise, our interaction-restored fliM mutants were identified after mutagenizing all of fliM. Our results are in general agreement with those of Brown et al. (2007). We have separately identified two FliM-binding sites on FliG. Two of our interaction-defective fliG mutations affect amino acid residues within the conserved hydrophobic patch; the others affect residues immediately adjacent to the hydrophobic patch or EHPQR motif (see Supplementary Fig. S3), suggesting that the binding domains are larger than these motifs, comprising, for example, hydrophobic residues adjacent to the EHPQR motif, hydrophobic regions on the side chains of two non-hydrophobic amino acid residues adjacent to the hydrophobic patch, and an additional adjacent hydrophobic amino acid found in E. colibut not in T. maritima. Our data suggest that the 'interior' high-affinity FliM-binding site of FliG is more likely to be filled first. We draw this conclusion because our mutants were all identified and characterized based on a direct assay for proteinprotein interaction that yields useful information about the relative affinities of protein complexes (Estojak et al., 1995). However, it is possible that other protein-protein interactions involving the switch could alter the relative occupancy of the two sites. In summary, our experiments complement and extend those of Brown et al. (2007) and lend additional support to their model.

\section{ACKNOWLEDGEMENTS}

We thank Andrew Kirsh for helpful comments on the manuscript. Special thanks go to David Blair for providing us with the coordinates for his model of E. coli FliM structure based on the CheC structure, for sharing his unpublished manuscript prior to submission, and for his encouraging comments, feedback and patience. Special thanks go to David DeRosiers for his permission to use his electron micrograph image of the basal body/C-ring complex. We also thank everybody who has shared plasmids, strains and other resources over the years. This work was supported by a grant from the National Science Foundation (MCB-0296079) to D.L.M. and S.E.P. R.M. was supported by a grant from the NIH RISE program (GM071638).

\section{REFERENCES}

Armstrong, J. B., Adler, J. \& Dahl, M. M. (1967). Nonchemotactic mutants of Escherichia coli. J Bacteriol 93, 390-398.

Bartlett, D. H. \& Matsumura, P. (1984). Identification of Escherichia coli region III flagellar gene products and description of two new flagellar genes. $J$ Bacteriol 160, 577-585.
Berg, H. C. (1988). A physicist looks at bacterial chemotaxis. Cold Spring Harb Symp Quant Biol 53, 1-9.

Blomfield, I. C., Vaughn, V., Rest, R. F. \& Eisenstein, B. I. (1991). Allelic exchange in Escherichia coli using the Bacillus subtilis sacB gene and a temperature-sensitive pSC101 replicon. Mol Microbiol 5, 1447-1457.

Brent, R. \& Finley, R. L., Jr (1997). Understanding gene and allele function with two-hybrid methods. Annu Rev Genet 31, 663-704.

Brown, P. N., Hill, C. P. \& Blair, D. F. (2002). Crystal structure of the middle and C-terminal domains of the flagellar rotor protein FliG. EMBO J 21, 3225-3234.

Brown, P. N., Mathews, M. A. A., Joss, L. A., Hill, C. P. \& Blair, D. F. (2005). Crystal structure of the flagellar rotor protein FliN from Thermotoga maritima. J Bacteriol 187, 2890-2902.

Brown, P. N., Terrazas, M., Paul, K. \& Blair, D. F. (2007). Mutational analysis of the flagellar protein FliG: sites of interaction with FliM and implications for organization of the switch complex. J Bacteriol 189, 305-312.

Chien, C.-T., Bartel, P. L., Sternglanz, R. \& Fields, S. (1991). The twohybrid system: a method to identify and clone genes for proteins that interact with a protein of interest. Proc Natl Acad Sci U S A 88, 9578-9582.

Estojak, J., Brent, R. \& Golemis, E. A. (1995). Correlation of twohybrid affinity data with in vitro measurements. Mol Cell Biol 15, 5820-5829.

Fields, S. \& Song, O.-K. (1989). A novel genetic system to detect protein-protein interactions. Nature 340, 245-246.

Francis, N. R., Irikura, V. M., Yamaguchi, S., DeRosier, D. J. \& Macnab, R. M. (1992). Localization of the Salmonella typhimurium flagellar switch protein FliG to the cytoplasmic M-ring face of the basal body. Proc Natl Acad Sci U S A 89, 6304-6308.

Francis, N. R., Sosinsky, G. E., Thomas, D. \& DeRosier, D. J. (1994). Isolation, characterization and structure of bacterial flagellar motors containing the switch complex. J Mol Biol 235, 1261-1270.

Gill, G. \& Ptashne, M. (1987). Mutants of GAL4 protein altered in activation function. Cell 51, 121-126.

Gillen, K. L. \& Hughes, K. T. (1991). Negative regulatory loci coupling flagellin synthesis to flagellar assembly in Salmonella typhimurium. J Bacteriol 173, 6453-6459.

González-Pedrajo, B., Minamino, T., Kihara, M. \& Namba, K. (2006). Interactions between $\mathrm{C}$ ring proteins and export apparatus components: a possible mechanism for facilitating type III protein export. Mol Microbiol 60, 984-998.

Grünenfelder, B., Gehrig, S. \& Jenal, U. (2003). Role of the cytoplasmic $\mathrm{C}$ terminus of the FliF motor protein in flagellar assembly and rotation. J Bacteriol 185, 1624-1633.

Heimbrook, M. E., Wang, W. L. L. \& Campbell, G. (1989). Staining bacterial flagella easily. J Clin Microbiol 27, 2612-2615.

Irikura, V. M., Kihara, M., Yamaguchi, S., Sockett, H. \& Macnab, R. M. (1993). Salmonella typhimurium fliG and fliN mutations causing defects in assembly, rotation, and switching of the flagellar motor. J Bacteriol 175, 802-810.

Jarvik, J. \& Botstein, D. (1975). Conditional-lethal mutations that suppress genetic defects in morphogenesis by altering structural proteins. Proc Natl Acad Sci U S A 72, 2738-2742.

Jones, C. J., Macnab, R. M., Okino, H. \& Aizawa, S. (1990). Stoichiometric analysis of the flagellar hook-(basal-body) complex of Salmonella typhimurium. J Mol Biol 212, 377-387.

Khan, I. H., Reese, T. S. \& Khan, S. (1992). The cytoplasmic component of the bacterial flagellar motor. Proc Natl Acad Sci U S A 89, 5956-5960. 
Khan, S., Zhao, R. \& Reese, T. S. (1998). Architectural features of the Salmonella typhimurium flagellar motor switch revealed by disrupted C-rings. J Struct Biol 122, 311-319.

Kihara, M., Miller, G. U. \& Macnab, R. M. (2000). Deletion analysis of the flagellar switch protein FliG of Salmonella. J Bacteriol 182, 3022-3028.

Kutsukake, K., Ohya, Y. \& lino, T. (1990). Transcriptional analysis of the flagellar regulon in Salmonella typhimurium. J Bacteriol 172, 741-747.

Lloyd, S. A., Tang, H., Wang, X., Billings, S. \& Blair, D. F. (1996). Torque generation in the flagellar motor of Escherichia coli: evidence of a direct role for FliG but not for FliM or FliN. J Bacteriol 178, 223-231.

Lloyd, S. A., Whitby, F. G., Blair, D. F. \& Hill, C. P. (1999). Structure of the C-terminal domain of FliG, a component of the rotor in the bacterial flagellar motor. Nature 400, 472-475.

Magariyama, Y., Yamaguchi, S. \& Aizawa, S. (1990). Genetic and behavioral analysis of flagellar switch mutants of Salmonella typhimurium. J Bacteriol 172, 4359-4369.

Marykwas, D. L. \& Berg, H. C. (1996). A mutational analysis of the interaction between FliG and FliM, two components of the flagellar motor of Escherichia coli. J Bacteriol 178, 1289-1294.

Marykwas, D. L. \& Passmore, S. E. (1995). Mapping by multifragment cloning in vivo. Proc Natl Acad Sci U S A 92, 11701-11705.

Marykwas, D. L., Schmidt, S. A. \& Berg, H. C. (1996). Interacting components of the flagellar motor of Escherichia coli revealed by the two-hybrid system in yeast. J Mol Biol 256, 564-576.

Mathews, M. A. A., Tang, H. L. \& Blair, D. F. (1998). Domain analysis of the FliM protein of Escherichia coli. J Bacteriol 180, 5580-5590.

Miller, J. H. (1992). A Short Course in Bacterial Genetics, a Laboratory Manual for Escherichia coli and Related Bacteria. Cold Spring Harbor, NY: Cold Spring Harbor Press.

Oosawa, K., Ueno, T. \& Aizawa, S.-I. (1994). Overproduction of the bacterial flagellar switch complex proteins and their interactions with the MS ring complex in vitro. J Bacteriol 176, 3683-3691.

Park, S. Y., Chao, X., Gonzalez-Bonet, G., Beel, B. D., Bilwes, A. M. \& Crane, B. R. (2004). Structure and function of an unusual family of protein phosphatases: the bacterial chemotaxis proteins $\mathrm{CheC}$ and CheX. Mol Cell 16, 563-574.

Park, S. Y., Lowder, B., Bilwes, A. M., Blair, D. F. \& Crane, B. R. (2006). Structure of FliM provides insight into assembly of the switch complex in the bacterial flagella motor. Proc Natl Acad Sci U S A 103, 11886-11891.

Parkinson, J. S., Parker, S. R., Talbert, P. B. \& Houts, S. E. (1983). Interactions between chemotaxis genes and flagellar genes in Escherichia coli. J Bacteriol 155, 265-274.

Sandrock, T. M., O’Dell, J. L. \& Adams, A. E. M. (1997). Allele-specific suppression by formation of new protein-protein interactions in yeast. Genetics 147, 1635-1642.

Schwede, T., Kopp, J., Guex, N. \& Peitsch, M. C. (2003). SWISSMODEL: an automated protein homology-modeling server. Nucleic Acids Res 31, 3381-3385.

Scott, W. R. P., Hünenberger, P. H., Tironi, I. G., Mark, A. E., Billeter, S. R., Fennen, J., Torda, A. E., Huber, T., Krüger, P. \& van Gunsteren, W. F. (1999). The GROMOS biomolecular simulation program package. J Phys Chem A 103, 3596-3607.

Silverman, M. \& Simon, S. (1974). Flagellar rotation and the mechanism of bacterial motility. Nature 249, 73-74.

Sockett, H., Yamaguchi, S., Kihara, M., Irikura, V. M. \& Macnab, R. M. (1992). Molecular analysis of the flagellar switch protein FliM of Salmonella typhimurium. J Bacteriol 174, 793-806.

Sosinsky, G. E., Francis, N. R., DeRosier, D. J., Wall, J. S., Simon, M. N. \& Hainfeld, J. (1992). Mass determination and estimation of subunit stoichiometry of the bacterial hook-basal body flagellar complex of
Salmonella typhimurium by scanning transmission electron microscopy. Proc Natl Acad Sci U S A 89, 4801-4805.

Sujatha, S. \& Chatterji, D. (2000). Understanding protein-protein interactions by genetic suppression. J Genet 79, 125-129.

Suzuki, H., Yonekura, K. \& Namba, K. (2004). Structure of the rotor of the bacterial flagellar motor revealed by electron cryomicroscopy and single-particle image analysis. J Mol Biol 337, 105-113.

Tang, H. \& Blair, D. F. (1995). Regulated underexpression of the FliM protein of Escherichia coli and evidence for a location in the flagellar motor distinct from the MotA/MotB torque generators. J Bacteriol 177, 3485-3495.

Tang, H., Billings, S., Wang, X., Sharp, L. \& Blair, D. F. (1995). Regulated underexpression and overexpression of the FliN protein of Escherichia coli and evidence for an interaction between FliN and FliM in the flagellar motor. J Bacteriol 177, 3496-3503.

Tang, H., Braun, T. F. \& Blair, D. F. (1996). Motility protein complexes in the bacterial flagellar motor. J Mol Biol 261, 209-221.

Thomas, D. R., Morgan, D. G. \& DeRosier, D. J. (1999). Rotational symmetry of the $\mathrm{C}$ ring and a mechanism for the flagellar rotary motor. Proc Natl Acad Sci U S A 96, 10134-10139.

Thomas, D., Morgan, D. G. \& DeRosier, D. J. (2001). Structures of bacterial flagellar motors from two FliF-FliG gene fusion mutants. J Bacteriol 183, 6404-6412.

Thomas, D. R., Francis, N. R., Xu, C. \& DeRosier, D. J. (2006). The three-dimensional structure of the flagellar rotor from a clockwiselocked mutant of Salmonella enterica serovar Typhimurium. J Bacteriol 188, 7039-7048.

Togashi, F., Yamaguchi, S., Kihara, M., Aizawa, S.-I. \& Macnab, R. M. (1997). An extreme clockwise switch bias mutation in fliG of Salmonella typhimurium and its suppression by slow-motile mutations in motA and motB. J Bacteriol 179, 2994-3003.

Toker, A. S. \& Macnab, R. M. (1997). Distinct regions of bacterial flagellar switch protein FliM interact with FliG, FliN and CheY. J Mol Biol 273, 623-634.

Toker, A. S., Kihara, M. \& Macnab, R. M. (1996). Deletion analysis of the FliM flagellar switch protein of Salmonella typhimurium. J Bacteriol 178, 7069-7079.

Yamaguchi, S., Aizawa, S., Kihara, M., Isomura, M., Jones, C. J. \& Macnab, R. M. (1986a). Genetic evidence for a switching and energytransducing complex in the flagellar motor of Salmonella typhimurium. J Bacteriol 168, 1172-1179.

Yamaguchi, S., Fujita, H., Ishihara, A., Aizawa, S. \& Macnab, R. M. (1986b). Subdivision of flagellar genes of Salmonella typhimurium into regions responsible for assembly, rotation, and switching. J Bacteriol 166, 187-193.

Yolov, A. A. \& Shabarova, Z. A. (1990). Constructing DNA by polymerase recombination. Nucleic Acids Res 18, 3983-3986.

Young, H. S., Dang, H., Lai, Y., DeRosier, D. J. \& Khan, S. (2003). Variable symmetry in Salmonella typhimurium flagellar motors. Biophys J 84, 571-577.

Zhao, R., Schuster, S. C. \& Khan, S. (1995). Structural effects of mutations in Salmonella typhimurium flagellar switch complex. J Mol Biol 251, 400-412.

Zhao, R., Amsler, C. D., Matsumura, P. \& Khan, S. (1996a). FliG and FliM distribution in the Salmonella typhimurium cell and flagellar basal bodies. J Bacteriol 178, 258-265.

Zhao, R., Pathak, N., Jaffe, H., Reese, T. S. \& Khan, S. (1996b). FliN is a major structural protein of the C-ring in the Salmonella typhimurium flagellar basal body. J Mol Biol 261, 195-208.

Edited by: J. G. Shaw 